\title{
Figural goodness effects in perception and memory
}

\author{
ROBIN MERMELSTEIN and WILLIAM BANKS \\ Pomona College, Claremont, California 91711 \\ and \\ WILLIAM PRINZMETAL \\ Claremont Graduate School, Claremont, California 91711
}

\begin{abstract}
There is an apparent contradiction concerning configurational effects in visual information processing. Some studies have shown that when an array is organized into a "good" or unitary Gestalt, analysis of a single part of it is facilitated, while others have shown "good" arrays to impede search for a part. The three experiments reported here support the proposition that goodness of form can facilitate performance when memory is used, but that goodness impairs strictly perceptual search for a part of an array. These experiments compare detection of a single feature in faces (unitary figures) and nonfaces. They show that when the face or nonface is presented before the target feature (and must be held in memory), performance is better for faces than for nonfaces. When the target is presented before the face or nonface and perceptual search is required, faces give worse performance than nonfaces. Implications for perceptual phenomena, including the object-superiority and wordsuperiority effects, are discussed.
\end{abstract}

The purpose of this series of experiments is to examine one possible resolution of a conflict in the visual information processing literature concerning Gestalt-like effects of stimulus configuration. While configurational effects on processing part of an array are well established, some studies have found that a part of a good form is detected more easily than a part of a bad form (Homa, Haver, \& Schwartz, 1976; Pomerantz, Sager, \& Stoever, 1977; Weisstein \& Harris, 1974; Williams \& Weisstein, 1978; Wormersley, 1977), and others have found that a good form hinders detection of one of its constituent parts (Banks \& Prinzmetal, 1976; Millspaugh, 1978; Prinzmetal \& Banks, 1977). The present research demonstrates that both superiority and inferiority effects of good context (i.e., a facilitation and a hindrance in performance) can occur with the same stimuli when the degree of memory component involved in the experiment is varied. These experiments show that in a task that depends on memory for the configuration, having the array configured as a good

This research was supported partly by National Science Foundation Grant BNS 78-17442 and partly by NIH Grant 1 RO1 MH33279. 01, both to W. P. Banks. We thank Jim Pomerantz for his valuable editorial comments on a draft of this paper. R. Mermelstein is now at the Department of Psychology, University of Oregon, Eugene, Oregon 97403. W. Prinzmetal is now at the Department of Psychology, University of British Columbia, Vancouver, B. C., Canada. Requests for reprints should be sent to William P. Banks, Department of Psychology, Pomona College, Claremont, California 91711. form helps detection of a constituent part, but in a task that requires perceptual analysis of the configuration, a good form hinders detection of a constituent part. These experiments all use an array configured like a cartoon of a human face as a good form and a scrambled, nonfacelike array as a bad form. We are not, however, studying face perception per se, but intend that our conclusions apply beyond face stimuli.

Weisstein and Harris' (1974) study is generally considered a demonstration that array goodness can facilitate analysis of parts. In their experiment, subjects had to identify one of four diagonal line segments that differed in orientation and location relative to a fixation point. On each trial, one of the four target lines, together with one of several context patterns, was flashed briefly on a CRT screen, resulting in compound patterns. The important variable was the context in which the target was presented: the target was presented alone, combined with two overlapping squares to form a unitary three-dimensional figure (good context), or with a meaningless array of lines (poor context). Weisstein and Harris found that subjects identified the diagonal target line better when it was part of a unitary three-dimensional object than when it was part of the less well-structured pattern. They named this finding the "object superiority effect," which is reminiscent of the "word superiority effect"' (Reicher, 1969; Smith \& Haviland, 1972).

While the object superiority effect appears to be prima facie evidence for a configurational effect on 
the perceptual analysis of an array for a part, a case can be made for a strong memory component. In the unitary figures, the target lines formed four distinctive and identifiable figures, while in the bad forms they did not. Subjects could, with relative ease, memorize each unitary context and associate each whole array with the appropriate response (a, b, c, or d). Memorization of the random configurations in the poor contexts, however, would not be easy, and even if memorized by the subjects, they would be harder to recognize since the poor contexts are not as distinguishable from each other as the unitary forms. When presented with the poor context, the subject must either rely on a faulty memory of the whole figure or actually search for the target. Thus, the object superiority effect may be a memory advantage in recognizability as a whole for Gestalts rather than an advantage in perceptual analysis of a part for Gestalts.

We emphasize that the object (or Gestalt) superiority effect is still a configural effect by this interpretation, but the facilitation comes through improved memorability of a whole rather than through improved analysis for the part. We also emphasize that our explanation depends on perceptual differences between the good and bad forms, as well as on differences in memory. The difference between our interpretation and Weisstein and Harris' lies in the process by which performance is better for good than for bad configurations. We say that perceptual search for the target is not facilitated by organization, but rather that the "recognizability" of the array as a whole is. By our explanation, an element would be harder to see in a good than a bad form in a strictly perceptual task in which the overall appearance of the array could not be a factor in processing, but analysis of the array for the component feature was required. In fact, Pomerantz and Sager (cited in Pomerantz, in press, vide circa Figure 18) have shown just this, that counting of diagonal target lines in stimuli like Weisstein and Harris' is slower with the unitary configuration than with either the randomline configuration or the stimuli with no context lines at all.

Homa, Haver, and Schwartz (1976) also found what seems to be evidence that a unitary form can facilitate the perception of its constituent parts. Their procedure also places demands on memory, but in a different way than Weisstein and Harris' does. Homa et al.'s stimuli consisted of schematic faces, scrambled faces, and single feature faces. One of these three stimulus types was tachistoscopically presented, followed by a 2 -sec visual noise mask and then by a forced-choice test of one of three features: a pair of eyes, a nose, or a mouth. Following the offset of the mask, the subject looked out of the tachistoscope, and was tested on one of the three types of facial feature. The subject was shown a card with five variations of the feature tested on that trial and had to decide which one of those five variations had appeared in the stimulus figure he or she had just seen. Homa et al. found that subjects were more accurate at detecting a feature when it was part of a face than when it was part of a scrambled face, but accuracy was best of all when no context, only a single feature, had been shown. Homa et al. concluded that the perceptibility of the constituent parts of a stimulus configuration was better when the parts were arranged within a well-defined form than when they were put in a bad form.

In this case, easier encoding of faces than of scrambled faces and differential rates of memory loss during the retention interval may account for the "facial superiority effect." On any given trial, the subjects did not know which feature would be tested, and had to remember all three, and thus the entire face, during the lapse of several seconds between presentation of the figure and presentation of the feature. If Gestalts (faces) are easier to encode and retain than noncoherent forms (scrambled faces), then Homa et al.'s facial superiority effect is actually a memory advantage for Gestalts rather than a perceptual advantage. Evidence for an advantage in encoding (Cattell, 1886; Freeman, 1916) and retention (Attneave, 1954) for good forms is quite old. This is not the place to present an exhaustive review of the literature on the matter, but we can point out that the modern literature supports a facilitation by good form in both remembering patterns and in encoding or recognizing patterns (Checkosky \& Whitlock, 1973; Clement \& Varnadoe, 1967; Clement \& Weiman, 1970; Garner, 1974; Lappin, Snyder, \& Blackburn, 1971; Royer, 1971; Yin, 1969).

In general, tasks in which good forms have hindered perceptual analysis of a part have not had a strong memory component. Banks and Prinzmetal (1976) and Prinzmetal and Banks (1977) varied the perceptual clustering of target and noise in arrays presented for detection of a target. In Banks and Prinzmetal (1976), either the target was perceptually grouped with the noise elements in the array or else it was not perceptually grouped with the noise elements, but was separate from them. Banks and Prinzmetal found that the target was detected more poorly if it was part of a perceptual Gestalt than if it was not. In another series of experiments, Prinzmetal and Banks (1977) found that grouping of a target with noise by the principle of good continuation degraded detection performance relative to a condition in which the target was not so grouped. Millspaugh (1978) examined how pattern goodness affects performance in a samedifferent task and found, like Banks and Prinzmetal, an impairment in performance for good configurations as compared to bad configurations. 
Pomerantz et al. (1977; see also Pomerantz, in press) conducted a series of experiments that do not have any apparent dependence on memory but that show better target detection in good or organized arrays than bad ones. However, in these studies, in the cases where target detection was facilitated, the targets were lines or shapes that combined with the context lines to create new, easily recognizable forms. Pomerantz et al: suggest that their facilitatory contexts work not by improving perceptual analysis for the part, but rather by creating emergent features from the combination of the context and target. It is, then, the emergent features that subjects detect, not the component part. If this is true, Pomerantz et al.'s suggestion is complementary to ours in that together they should account for all cases of contextual interference and facilitation in detection. To put it briefly, detection of a target in a good or unitized array will be easier than detection of a target in a nonunitized array either if the task depends on memory such that the superior memory for the good array can help performance or if the target and context combine to make emergent forms that can be used to mediate performance with the good arrays but not the bad. If neither of these cases holds, a "camouflaging" effect will be found whereby perceptual analysis of a part will be harder in a good form than in a bad form. But for this synthesis of the two factors to be entertained, we must first establish the credibility of the effect of memory in detection, and that is the purpose of this article.

The three experiments reported here explicitly test whether processing a part of an array is facilitated by figural goodness when the array must be held in memory and is impaired by figural goodness when the array is presented for perceptual inspection. Experiment 1 is similar to the Homa et al. (1976) experiments that obtained a facilitation of performance for unitary stimuli (faces), but in this experiment the memory requirements that were in the Homa et al. experiment are largely eliminated. Here the subject searches a face or nonface stimulus for a target and is timed while searching the stimulus for the target. There is no requirement that the face be held in memory while the subject waits for the target. Since the task depends on perceptual analysis, we predicted that the target will be harder to find in the unitary form (scrambled face), and our prediction was confirmed. The second experiment compares the memory and perceptual components directly in a single within-subject experiment by either presenting the target before the face, in which case the task is one of visual search for the target in the face, or presenting the target after the face, in which case it is a memory search task. The results replicate Experiment 1 for the visual search task, show a face superiority effect for the memory task, and show the predicted interaction between stimulus type (face vs. nonface) and task (memory vs. perception). However, the face superiority effect in the memory condition by itself was not reliable, possibly because the task was too easy and subjects did not always need to rely on the organizational superiority of the faces to perform the task. Experiment 3 uses a more difficult task than Experiment 2 and shows that the Homa et al. effect can be replicated reliably with our stimuli. Experiment 3 also shows that the Homa et al. effect replicates for our stimuli with error rates as well as RT. This replication is important because Experiments 1 and 2 use RT while the Homa et al. study used error rate as the dependent variable.

These experiments used faces as the good or unitized forms for several reasons. First, Homa et al. demonstrated a robust superiority effect for faces, one that seems reasonable to attribute to some emergent Gestalt property of the face-like configuration (Yin, 1969). A second reason for choosing faces for our stimuli derives from a possible alternative resolution of the issue over whether good array organization facilitates or degrades performance. As it happens, those studies that show a facilitation of detection by array goodness used organizational principles that result in the depiction of meaningful or familiar objects such as planes oriented in three dimensions (Weisstein \& Harris, 1974), simple geometric shapes (Pomerantz et al., 1977), or real-world scenes (Biederman, 1972). On the other hand, the studies that have shown performance to be impaired by good array organization have used Gestalt principles such as good continuation (Prinzmetal \& Banks, 1977) that do not result in the depiction of familiar objects. Thus, it is possible that it is the pictorial, depictive nature of the organizational principle, or the familiarity of the object depicted, that determines whether organization will facilitate or impair performance. We therefore chose faces as stimuli, taking them to be depictive of a familiar class of object. Showing that performance was worse in some conditions with faces than with nonfaces would demonstrate that it was not simply the kind of organizational principle that determined whether organization would facilitate or degrade performance.

\section{EXPERIMENT 1}

The purpose of Experiment 1 is to examine the effects of context in a perceptual search task using schematic faces, scrambled faces, and single features as stimuli. Inverted versions of the same stimuli were also used. The inverted faces were used because in previous memory studies (Goldstein, 1965; Hochberg \& Galper, 1967; Yin, 1969), right-side-up faces were always recognized as a whole better than upsidedown faces. The scrambled and single-feature stimuli 
were inverted as a control for the inverted faces. Here we determine whether analysis of a part in a perceptual search task without contamination by memory requirements shows an advantage or a disadvantage for faces relative to the other sorts of stimuli.

\section{Method}

Materials and Design. The stimuli, faces, scrambled faces, and single features, consisted of hand-drawn features of eyes, noses, and mouths contained in oval outlines, as shown in Figure 1. For each face, there was a corresponding scrambled face consisting of the same three features, but with the eyes and mouth in inappropriate positions. There were four different types of eyes and four different types of mouths which were randomly paired together to make four unique combinations of eyes and mouth pairs, which formed the basis of each face or scrambled face. Figure 1 shows the four eye-mouth combinations. There were eight different noses, four symmetrical and four asymmetrical about the horizontal axis. Figures $1 \mathrm{~A}$ and $1 \mathrm{~B}$ contain examples of symmetrical noses (which look the same upside-down as right-side-up), and Figures 1C and 1D contain examples of asymmetrical noses (which do not look the same up-side-down). Each eye-mouth combination was paired with one symmetrical and one asymmetrical nose, giving eight faces and eight scrambled faces. The eight single-feature face stimuli consisted of the oval outline, but contained only one feature, the nose. Regardless of stimulus type,

\section{FACES}

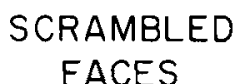

FACES

A
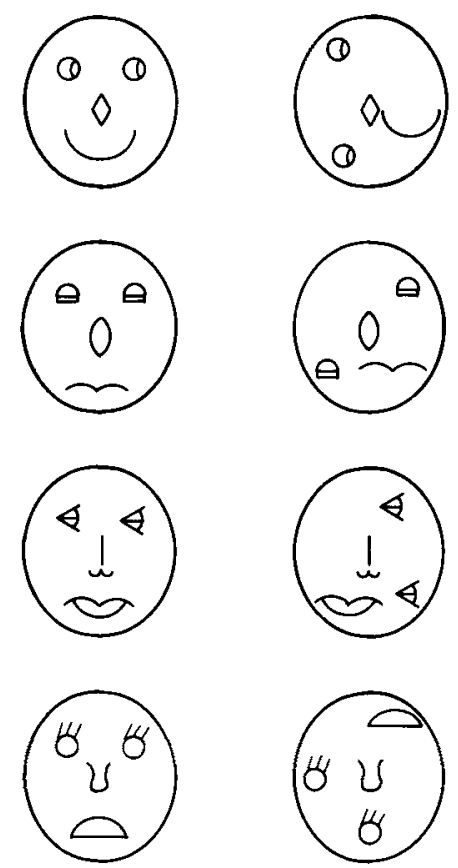

SINGLE FEATURES
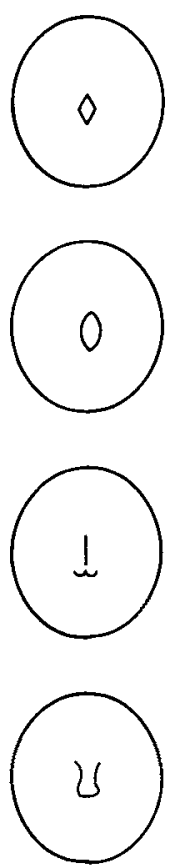

Figure 1. Examples of stimuli from all three experiments. The four sets of eyes and mouths shown combined (rows A-D under Faces and Scrambled Faces) always appeared together. No other eyes or mouths were used and no other combinations of these eyes and mouths were made. The nose, which was the target, was varied; four of the eight noses used are shown as examples. Noses in $A$ and $B$ are symmetrical and those in $C$ and $D$ are asymmetrical. the nose was always in its appropriate position and was always the target tested. For every right-side-up stimulus, there was a corresponding upside-down stimulus. There were 48 unique stimuli: 16 faces, 16 scrambled faces, and 16 single feature faces, with half of each upside down. The design was a within-subjects repeated-measures design with the following major variables: (1) stimulus type (face, scrambled face, single-feature face); (2) orientation (right side up, upside down); (3) symmetry of target (symmetrical, asymmetrical); (4) response (yes, no); and (5) eight blocks per session.

Procedure. Prior to the collection of data, each subject was shown a set of four targets, two asymmetrical noses and two symmetrical noses, which he or she was to memorize. This was the positive response set. Another set of four noses, two symmetrical and two asymmetrical noses, made up the negative response set. The possible combinations of noses in the two sets were counterbalanced across subjects. After memorization, the subject was told that he or she would be presented with one of three types of stimuli, a face, a scrambled face, or a single feature face, and was shown an example of each. The subject's task was to respond "yes" or "no" to each stimulus depending on whether one of the memorized targets was contained in that stimulus. If a target from the memory set appeared upside down, it was still considered a positive response. The subject was told to respond as quickly as possible and as accurately as possible. Responses were made by pressing one of two keys.

All stimuli were presented in an Iconix tachistoscope, at a viewing distance of $86 \mathrm{~cm}$, and subtended a visual angle of 2.6 $\times 2.3 \mathrm{deg}$. All fields of the tachistoscope had a luminance of about $20 \mathrm{~mL}$, and a subject was always looking at an illuminated field. The subjects saw a black fixation point for $500 \mathrm{msec}$, followed immediately by presentation of the stimulus. The reaction time (RT) was recorded from the onset of the stimulus, and the stimulus remained on until the subject responded. The subjects received 16 practice trials, chosen randomly from the stimulus cards. The data trials consisted of 8 blocks of 48 trials each, totaling 384 trials. Incorrect trials were repeated at the end of each block, and only correct RTs were recorded. The stimulus cards were shuffled between blocks, as well as between subjects.

Subjects. Six high school students, recruited through an advertisement in a local newspaper, served as subjects. Each received $\$ 2$ for the $1-\mathrm{h}$ experiment.

\section{Results and Discussion}

Table 1 shows the chief effects of interest, and it is clear that the face superiority effect is reversed in this perceptual task. The main effect of stimulus type was highly significant $[\mathrm{F}(2,10)=10.03, \mathrm{p}<.005]$. Subjects responded to single-feature faces the fastest $(\overline{\mathrm{X}}=649 \mathrm{msec})$. More important, subjects were faster at detecting the target when it was in a scrambled face than when it was in a face $(686$ vs. $743 \mathrm{msec})$. A $t$ test comparing the face and scrambled face stimuli was significant $[\mathrm{t}(10)=4.82, \mathrm{p}<.01]$.

The other variable of interest, orientation, was not significant $[F(1,5)<1.0]$, and there was no interaction between stimulus type and orientation $[\mathrm{F}(2,10)<1.0]$. As seen in Table 1, upside-down faces were not worse than right-side-up faces, as was the case in recognition memory studies (Goldstein, 1965; Hochberg \& Galper, 1967; Yin, 1969), but were actually slightly faster. Although there is no significant difference between upside-down faces and rightside-up faces, there appears to be a continuum of facedness on which upside-down faces lie between 
Table 1

Mean Reaction Time (in Milliseconds)

Performance in Experiment 1

\begin{tabular}{|c|c|c|c|}
\hline \multirow[b]{2}{*}{ Orientation } & \multicolumn{3}{|c|}{ Stimulus Type } \\
\hline & Face & $\begin{array}{c}\text { Scrambled } \\
\text { Face }\end{array}$ & $\begin{array}{l}\text { Single- } \\
\text { Feature } \\
\text { Face }\end{array}$ \\
\hline $\begin{array}{l}\text { Right Side Up } \\
\text { Upside Down }\end{array}$ & $\begin{array}{l}762 \\
724\end{array}$ & $\begin{array}{l}686 \\
686\end{array}$ & $\begin{array}{l}647 \\
652\end{array}$ \\
\hline Mean & 743 & 686 & 650 \\
\hline
\end{tabular}

the polar points of faces and scrambled faces. As one moves along this continuum from faces to scrambled faces, reaction times decrease. In very good accord with our hypothesis, the less meaningful or coherent the stimulus, the faster the response.

Two other main effects were significant: response $[F(1,5)=29.99, p<.005]$ and symmetry of target $[\mathrm{F}(1,5)=10.84, \mathrm{p}<.025]$. "Yes" responses were faster than "no" responses ( 672 vs. $725 \mathrm{msec}$ ). The subjects responded to asymmetrical targets $(\overline{\mathrm{X}}=$ $673 \mathrm{msec})$ faster than to symmetrical targets $(\bar{X}=$ $724 \mathrm{msec}$ ). One might have expected symmetrical targets to be faster than asymmetrical targets because when the latter are inverted, they look different from the target noses. These targets may have been more distinctive, though, than the symmetrical ones and thus identified faster.

Overall error rates were low, ranging from $.5 \%$ to $3.1 \%$, with a mean of $1.6 \%$ over subjects. The error rates for the three stimulus types were: faces, $2.8 \%$; scrambled faces, $3.1 \%$; and single-feature faces, $3.8 \%$. It is unlikely that the pattern of RTs was caused by the small overall speed-accuracy tradeoff because the tradeoff was found for only two of the six subjects. When the data from the two subjects are removed, the RT difference between the stimulus types actually increases. The mean RTs for the four subjects without a speed-accuracy tradeoff are: faces, $749 \mathrm{msec}$; scrambled faces, $674 \mathrm{msec}$; and single-feature faces, $640 \mathrm{msec}$. For the two subjects who had a speedaccuracy tradeoff, the pattern of results is the same: faces, $740 \mathrm{msec}$; scrambled faces, $698 \mathrm{msec}$; and single-feature faces, $660 \mathrm{msec}$.

Although the subjects had only to identify the nose, ard although the target was always in the same location regardless of stimulus type, subjects were faster with scrambled faces than with faces. The context of a good form had an inhibitory effect. This finding does not replicate the Homa et al. face superiority effect and suggests that our hypothesis is correct that goodness of form has different effects in perception and memory. Experiment 2 attempts a direct test of this hypothesis by comparing perceptual search of a visual array with search of a memorized array. Our hypothesis is really that there is an interaction between task (memory search vs. perceptual search) and goodness of form. Experiment 1 demonstrates only half of this interaction and must be compared with the Homa et al. research to support the conclusion. It would be better to use the same stimuli and show opposite effects for perception and memory; consequently, we performed Experiment 2.

\section{EXPERIMENT 2}

\section{Method}

The stimuli of Experiment 1 were used. A new variable, order of feature (target) presentation, was added to this experiment. In the perceptual search condition, the feature was presented before the figure on each trial. The feature, which was again always the nose, was briefly presented for $100 \mathrm{msec}$, followed by a 3-sec blank interval and then by presentation of one of the three stimulus types (face, scrambled face, or single-feature face). The subject's task was to respond by pressing the appropriate key to indicate whether the nose was the one in the figure he or she was viewing. RT was recorded from the onset of the figure, which remained on until the subject responded. In the memory search condition, the figure (face, scrambled face, or single-feature face) was presented before the feature. The figure was presented for $100 \mathrm{msec}$, followed by a 3-sec blank interval and then by presentation of the feature (always the nose). The subject's task was to respond with a keypress to indicate whether the probe nose was contained in the figure he or she had seen. RT was recorded from the onset of the probe nose, which remained on until the subject responded. In both conditions, the probe nose was always presented upright, although half of the figures were inverted. Between trials, the subject fixated on a black dot in the center of the visual field.

All subjects were tested under both conditions, and order of condition was counterbalanced across subjects. Half of the subjects received 4 blocks of 48 trials each for the memory condition first, followed by 4 blocks of 48 trials each for the perceptual condition; the other half of the subjects received the reverse order. Incorrect trials were repeated at the end of each block, and only correct responses were recorded. There were 16 practice trials before the first block of each condition. This was a within-subjects repeated-measures 2 by 3 by 2 by 2 by 2 by 4 factorial design. The variables were: (1) order of target presentation, (2) stimulus type, (3) orientation, (4) symmetry of target, (5) response, and (6) repetitions over blocks.

Subjects. The subjects were eight Claremont Colleges students, none of whom had participated in Experiment 1. Each received $\$ 4$ for participation in the 2 -h experiment.

\section{Results and Discussion}

Figure 2 shows the critical interaction between order of target presentation and stimulus type. The analysis of variance performed on the RTs found this interaction to be highly significant $[F(2,14)=10.82$, $\mathrm{p}<.005]$. When the target feature (nose) was presented before the figure (perceptual search), subjects responded to scrambled faces faster than to faces [t(7) $=8.96, p<.001]$, replicating the results of Experiment 1 . When the target feature was presented after the figure (memory search), however, the advantage for scrambled faces disappeared $[\mathrm{t}(7)=.67]$ and there was no difference between faces and scrambled faces. Overall, the main effect of stimulus type was significant $[\mathrm{F}(2,14)=11.32, \mathrm{p}<.005]$. Single-feature faces were the fastest $(\bar{X}=556 \mathrm{msec})$, and scrambled 


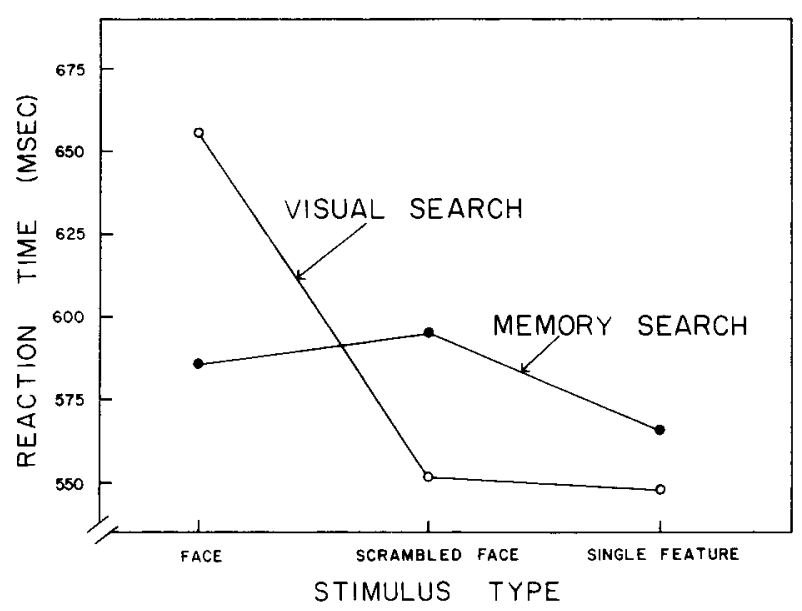

Figure 2. Reaction time as a function of stimulus type for both the visual search and the memory search conditions.

faces $(\overline{\mathrm{X}}=574 \mathrm{msec})$ were faster than faces $(\overline{\mathrm{X}}=$ $622 \mathrm{msec}$ ). Error rates in both conditions were low. In the perceptual search condition, subjects made the most errors on faces $(3.1 \%)$, followed by single-feature faces $(1.6 \%)$ and then by scrambled faces $(.8 \%)$. The error rates in the memory search condition were: faces, $.8 \%$; scrambled faces, $1.2 \%$; and single-feature faces, $1.9 \%$.

The interaction between orientation and symmetry of target was also significant $[\mathrm{F}(1,7)=7.26, \mathrm{p}<$ $.05]$. For upside-down stimuli, symmetrical targets were faster than asymmetrical targets (571 vs. $597 \mathrm{msec}$ ), but there was almost no difference in symmetry for right-side-up stimuli $(581 \mathrm{msec}$ for asymmetrical and $587 \mathrm{msec}$ for symmetrical targets). Why there is a decrease in reaction time with symmetrical targets for upside-down stimuli as compared to right-side-up stimuli seems inexplicable at this point. There was no significant three-way interaction between stimulus type, orientation, and symmetry $(\mathrm{F}<1.0)$.

There was a reliable interaction between response and order of target $[\mathrm{F}(1,7=6.91, \mathrm{p}<.05]$. In both conditions, "yes" responses were faster than "no" responses $[\mathrm{F}(1,7)=10.84, \mathrm{p}<.025]$. When the target came after the figure, though, the difference between the two responses became much greater than when the target came first (25-msec difference for target first vs. 87-msec difference for target second).

Although the predicted interaction between type of stimulus and type of task (memory search vs. perceptual search) was reliable, the face superiority effect found reliably by Homa et al. for the memory condition alone was small $(6 \mathrm{msec})$ and unreliable here. At least two factors may, however, have operated against our finding a strong memory effect. The first is subject strategies. In the 3-sec blank interval between presentation of the figure and presentation of the target feature, subjects had enough time to process the face or scrambled face, inspect the nose, and remember only the nose to compare with the test stimulus. As a result, the task may have been testing memory for only a single feature instead of memory for the whole stimulus. Experiment 3, therefore tests equally often with all the features-the eyes, the nose, and the mouth in order to increase the probability of obtaining a reliable face superiority effect in memory. It is important to obtain this effect to show that the differences between our results and Homa et al.'s (1976) come from the differences between perceptual and memory components and not some trivial difference between their stimuli or procedure and ours.

A second factor that might increase the facial superiority effect in memory is the presence of a pattern mask. McClelland (1978) studied the effects of a mask on the object superiority effect. His stimuli consisted of contexts similar to those of Weisstein and Harris (1974). McClelland found that when he used a patterned line mask, detection of lines in objects was reliably better than detection of lines in the poor contexts. When he used a blank mask, similar to the blank interval used in Experiment 2, however, there was no difference between detection of lines in objects and detection of lines in the poor contexts. In other words, the object superiority effect disappeared. Homa et al. (1976) also used a patterned mask in their experiment. Experiment 3 tests whether a patterned mask affects the size of a face superiority effect.

\section{EXPERIMENT 3}

\section{Method}

Materials and Design. The target-first condition was no longer investigated, and all subjects were tested under only the figurefirst, target-second (memory search) condition. In addition, singlefeature face stimuli were no longer used. The main variables of the design used were: (1) group (mask vs. no mask); (2) stimulus type (face vs. scrambled face); (3) orientation (right side up vs. upside down); (4) target (eyes, nose, and mouth); (5) response (yes, no). There were repeated measures on all variables except group. The difference between the two groups was the presence or lack of a visual noise mask in the interval between presentation of the figure and presentation of the feature. The subjects were randomly assigned to the two groups. The visual noise mask consisted of an oval outline twice the size of that used in the face and scrambled-face stimuli and contained a random arrangement and selection of all the possible target features--eyes, noses, and mouths. It contained a total of 16 features.

The same stimuli as in Experiment 2 were used with the addition of eight new target stimuli. The target stimuli consisted of the four different sets of eyes and the four different mouths that appeared in the figures. In addition, the eight target stimuli from Experiment 2, consisting of the eight different noses, were also used. There was, thus, a total of 32 stimulus figures (16 faces, 16 scrambled faces, with half of each upside down). For each figure, the subjects were tested twice on each feature on different trials, with one positive response and one negative response. Thus, a subject would see each figure six times ( 3 features by 2 responses), once in each of the six blocks of 32 trials each, or 
each figure once per block. The feature tested and response were counterbalanced both within blocks and across blocks. In any one block, the subjects responded to an approximately equal number of eyes, noses, and mouths (one feature was tested once less often per block because 32 does not divide evenly by 3 , but equally across blocks), and responded to an equal number of yeses and noes. All target features were presented upright in the center of a plain white background, with no other features or facial outline.

Procedure. The same apparatus was used as in the previous experiments. Subjects in Group 1 received the following order of presentation: fixation dot for $500 \mathrm{msec}$, followed by the figure, which was on for $100 \mathrm{msec}$, then by a 2-sec blank interval, and finally by the target feature. The target feature could be a pair of eyes, a nose, or a mouth. RT was recorded from the onset of the target feature, which remained on until the subject responded by pressing a key. The subjects in Group 2 received the same order, but with two modifications: the stimulus figure duration was increased to $200 \mathrm{msec}$ and was followed by the visual noise mask. Pilot work showed that a 200 -msec exposure duration in combination with the mask would give approximately the same overall performance as the 100 -msec, no-mask condition. For both groups, the subject's task was to respond "yes" or "no" as to whether the feature he or she saw second was contained in the figure he or she saw first. The subjects were given 16 practice trials.

Subjects. Sixteen Claremont Colleges students participated in the $1-h$ experiment. Each was paid $\$ 2$, and none had participated in either Experiment 1 or 2.

\section{Results}

Both RT and error rates were recorded for each subject, and separate analyses of variance were performed on the two dependent measures.

Reaction time. Overall, RTs were much longer in this experiment than in the previous two, with an overall mean of $1,284 \mathrm{msec}$. There were no significant main effects for the RT measures, although faces had a slight advantage over scrambled faces $(1,276 \mathrm{msec}$ for faces vs. 1,293 msec for scrambled faces).

There was one significant RT interaction between stimulus type and response $[\mathrm{F}(1,14)=5.26, \mathrm{p}<.05]$. This interaction is such that for faces, "no" responses were faster than "yes" responses; but for scrambled faces, the reverse was true: "yes" responses were faster than "no" responses.

Error rate. The error rates were a more sensitive measure than RTs in this experiment. The subjects made significantly more errors on scrambled faces than on faces $[F(1,14)=6.27, p<.05]$. Table 2 shows both error rates and RTs for faces and scrambled faces. Overall, error rates ranged from .146 to .375 .

Table 2

Mean Reaction Time (in Milliseconds) and Error Rates (in Percentages) in Experiment 3

\begin{tabular}{ccc} 
& \multicolumn{2}{c}{ Dependent Measure } \\
\cline { 2 - 3 } Stimulus & Reaction & Error \\
Type & Time & Rate \\
\hline Face & 1295 & 23.0 \\
Scrambled Face & 1301 & 27.6 \\
\hline
\end{tabular}

There was no reliable main effect of mask for either the $\mathrm{RT}(\mathrm{F}<1.0)$ or the error rates $[\mathrm{F}(1,14)=$ $4.41, \mathrm{p}<.10]$, although the group with the mask made slightly more errors than did the no-mask group (.287 vs. .219) and were also slightly slower $(1,275$ vs. $1,294 \mathrm{msec})$. Furthermore, there was no interaction between group and stimulus type $(\mathrm{F}<$ $1.0)$ for either measure. That is, the mask did not differentially affect faces and scrambled faces.

Error rates differed significantly across target features. Overall, subjects made the least errors on noses (.229) and fewer errors on eyes (.237) than on mouths (.294). The type of target also interacted with orientation. For right-side-up features, the subjects were most accurate on eyes, followed by noses, and then by mouths. For upside-down-features, however, the subjects were most accurate on noses, followed by eyes, and then by mouths.

Although all three features were tested, only the nose was controlled for spatial location uncertainty across stimulus type; it was always in its appropriate position regardless of stimulus type. The crucial test for a facial superiority effect in this experiment lies in a separate analysis on just the nose data. The subjects made significantly more errors on noses in scrambled faces than on noses in faces (.240 vs. .217) $(\mathrm{p}<.05$ by the sign test $)$.

\section{GENERAL DISCUSSION}

The results of the present experiments support the conclusion that goodness of form that facilitates performance in detecting a part of a memorized array may degrade performance in perceptual search of the same array. As the present research shows, it is possible to obtain both facilitation and interference in performance using the same stimuli. If the task requires perceptual analysis of the array, good forms can hinder detection of one of their parts, as though they camouflaged it. On the other hand, if the task requires memory of the array, the same good forms can help detection of a part, possibly because unitary forms are remembered better than disorganized ones or because "camouflaging" does not operate in encoded memory in the same way as in perception.

Considering this study together with previous works from this laboratory (Banks \& Prinzmetal, 1976; Prinzmetal \& Banks, 1977) and with work by Pomerantz and his colleagues (Pomerantz, in press; Pomerantz et al., 1977), we can make some generalizations that may resolve the issue over whether goodness of an array helps or hinders performance. Good array organization can facilitate performance relative to bad organization in two ways: first, if the array has to be stored in memory for performance of the task; and, second, if the different target elements somehow combine with other array elements to 
create different emergent forms that are correlated with the correct response associated with the target element in each array. The basis of the first principle, the memory advantage for Gestalts, is not clear at this point. It could be a learning effect or a retrieval effect. In a given short presentation interval, Gestalts may be encoded or integrated ("learned") faster than noncoherent forms. It may also be easier to access information from a memory trace based on a good form than on a bad form.

With faces, emergent aspects would be of a different nature from those in the Pomerantz et al. (1977) experiment. Instead of the geometrical shape found in the Pomerantz et al. study, the emergent characteristics would be more in the nature of a high-level code or overall character that subjects could use in addition to individual features. When tested with a single feature, subjects could match the feature to the overall impression in addition to comparing it with whatever information they may have about the parts. For example, a subject might get an impression of a happy face, but not have specific information about individual features. If the subject were then presented with a frowning mouth as the target feature, the subject could respond "no" even though he or she had forgotten the particular mouth used. For scrambled faces, however, there would be no overall impression about the figure, and subjects would base their responses entirely on the limited information they had about the individual features. We should emphasize that we have no way to predict when an emergent feature will be created by an arrangement of lines on a stimulus, either for the geometric shapes of Pomerantz' work or for faces in the present study. We do know how to create some forms, such as faces, that will behave as Gestalts, and we can use them as stimuli in experiments to study the processing of wholes, but this does not mean that we have a theoretical understanding of how parts combine to make a whole in some configurations and not in others (cf. Banks \& Prinzmetal, 1976, pp. 366-367).

Good organization of an array can interfere with detection of a component part if there is no facilitation through a memory component or emergent characteristics, and the subject must therefore search through the array for the target. There are several possible mechanisms by which the camouflaging effect of a good form could work. First, the processing strategies used for Gestalts may be different from those used for noncoherent forms. Gestalts, such as faces, are, by this explanation first processed holistically and then searched for a distinguishing feature. Information from the Gestalt comes in as a whole unit because the individual features of the Gestalt interact with each other to form a well-integrated pattern. However, the individual features of the pattern are subordinate to the whole, and the target features are therefore hidden or camouflaged in it.
A directed search for the target or a perceptual reorganization is then necessary to pull out the features needed for the decision. A definite match or no match could then be made when the separate and individual features are isolated. For noncoherent forms, such as a scrambled face, there is no organized or well-defined whole that dominates perception and hides the features. Instead, the search and comparison of individual features can begin immediately. This explanation is similar to that of Banks and Prinzmetal (1976), which employed a perceptual "parser." This explanation is also similar to the "sealed-channel" hypothesis of Pomerantz (in press).

The other explanation of the perceptual goodness effects is attentional. Although the subjects had to identify only one feature, and this feature was always in the same location regardless of stimulus type, the subjects could not ignore the fact that they were seeing a face. In other words, the presence of an organized whole pattern forced their attention temporarily to the whole, instead of to the individual features. The overriding presence of the Gestalt thus slowed the subjects' detection of features. With scrambled faces, however, the subject's attention is directed immediately to the individual units. Thus, the impairment in performance for faces may be due to a misappropriation of attentional capacities.

These experiments invite comparison with recent experiments on the word superiority effect (in which letters are recognized better in the context of words than either in nonwords or alone; cf. Reicher, 1969). Recent treatments of the word superiority effect (Estes, 1975; Massaro, 1973; Thompson \& Massaro, 1973) have attributed the advantage of words to sophisticated guessing strategies or to memory factors. Thompson and Massaro (1973) manipulated the time at which a subject was given the response alternatives. When the letter alternatives followed the word presentation, Thompson and Massaro found an advantage for words over single letters. When the subject received the response alternatives before the presentation of words, however, the word superiority effect disappeared. Bjork and Estes (1973) and Johnston and McClelland (1974) found a similar result. Thompson and Massaro concluded that when the alternatives are second, the subject synthesizes the critical letter prior to the onset of alternatives. The subject can then use sophisticated guessing strategies to arrive at a decision. These results can be compared to those of Experiments 1 and 2. When the critical feature is known before presentation of the figure, there is no facial superiority effect, but rather, an advantage for scrambled faces. In the memory condition of Experiment 2 and in Experiment 3, when the feature is presented after the figure, sophisticated guessing strategies can come into play, and a facial superiority effect is found.

Massaro (1973) also proposed a memory explana- 
tion for previous word superiority effects, for example, that found by Smith and Haviland (1972). The subjects in Smith and Haviland's study had to look at a choice card that was presented outside the tachistoscope. Massaro suggests that there was differential forgetting during this time for words and nonwords. The subjects might recognize the letters equally well on the word and nonword trials, but to remember them they must rehearse them until they see the probe card outside the tachistoscope. In the word condition, the subjects need only rehearse one word; but in the nonword condition, they must rehearse three separate letters, and the word superiority effect could thus be a memory advantage for words and not a perceptual advantage.

Massaro's (1973) explanation of the word superiority effect is strikingly similar to our explanation of Homa et al.'s (1976) facial superiority effect. Our study shows that the facial superiority effect depends on a memory component in the task; without it, there is a facial inferiority effect. The mechanism of the different effects in perception and memory is, however, not yet known.

\section{REFERENCES}

Attneave, F. Some informational aspects of visual perception. Psychological Review, 1954, 61, 183-193.

Banks, W. P., \& Prinzmetal, W. Configurational effects in visual information processing. Perception \& Psychophysics, 1976, 19, 361-367.

Biederman, I. Perceiving real-world scenes. Science, 1972, 177, $77-80$.

BJork, E. L., \& Estes, W. K. Letter identification in relation to linguistic context and masking conditions. Memory \& Cognition, 1973, 1, 217-233.

CatTell, J. M. The time taken by cerebral operations. Mind, $1886,11,524-538$.

Checkosky, S. F., \& Whitlock, D. The effects of pattern goodness on recognition time in a memory search task. Journal of Experimental Psychology, 1973, 100, 341-348.

Clement, D. E., \& Varnadoe, K. W. Pattern uncertainty and the discrimination of visual patterns. Perception \& Psychophysics, 1967, 2, 427-431.

Clement, D. E., \& Weiman, C. F. R. Instructions, strategies, and pattern uncertainty in a visual discrimination task. Perception \& Psychophysics, 1970, 7, 333-336.

GaRnER, W. R. The processing of information and structure. New York: Erlbaum, 1974.

Estes, W. K. The locus of inferential and perceptual processes in letter identification. Journal of Experimental Psychology: General, 1975, 104, 122-145.
Freeman, F. N. Experimental education. Boston: Houghton Mifflin, 1916.

GoLDSTEIN, A. Learning of inverted and normally oriented faces in children and adults. Psychonomic Science, 1965, 3, 447-448.

Hochberg, J., \& Galper, R. E. Recognition of faces: I. An exploratory study. Psychonomic Science, 1967, 9, 619-620.

Homa, D., Haver, B., \& Schwartz, T. Perceptibility of schematic face stimuli: Evidence for a perceptual gestalt. Memory \& Cognition, 1976, 4, 176-185.

Johnston, J. C., \& MCClelland, J. L. Perception of letters in words: Seek not and ye shall find. Science, 1974, 184, 11921194.

Lappin, J. S., Snyder, C. R., \& Blackburn, C. The encoding of perceptual information in the organization of individual stimulus patterns. Perception \& Psychophysics, 1971, 10, 123-128.

Massaro, D. W. Perception of letters, words, and nonwords. Journal of Experimental Psychology, 1973, 100, 349-353.

McClelland, J. L. Perception and masking of wholes and parts. Journal of Experimental Psychology: Human Perception and Performance, 1978, 4, 210-223.

Millspaugh, J. R. Effects of array organization on same-different judgments. Perception \& Psychophysics, 1978, 23, 27-35.

Pomerantz, J. R. Perceptual organization in information processing. In M. Kubovy \& J. R. Pomerantz (Eds.), Perceptual organization. New York: Erlbaum, in press.

Pomerantz, J. R., Sager, L. C., \& Stoever, R. J. Perception of wholes and of their component parts: Some configural superiority effects. Journal of Experimental Psychology: Human Perception and Performance, 1977, 3, 422-435.

Prinzmetal, W., \& Banks, W. P. Good continuation affects visual detection. Perception \& Psychophysics, 1977, 21, 389-395.

Reicher, G. M. Perceptual recognition as a function of meaningfulness of stimulus materials. Journal of Experimental Psychology, 1969, 81, 275-280.

ROYER, F. L. Spatial orientational and figural information in free recall of visual figures. Journal of Experimental Psychology, $1971,91,326-332$.

Smith, E. E., \& Haviland, S. E. Why words are perceived more accurately than nonwords. Journal of Experimental Psychology, 1972, 92, 59-64.

Thompson, M. C., \& Massaro, D. W. Visual information and redundancy in reading. Journal of Experimental Psychology, $1973,98,49-54$.

Weisstein, N., \& Harris, C. S. Visual detection of line segments: An object superiority effect. Science, 1974, 186, 752 755.

Williams, A., \& Weisstein, N. Line segments are perceived better in a coherent context than alone: An object-line effect in visual perception. Memory \& Cognition, 1978, 6, 85-90.

Wormerstey, M. A. Contextual effect in feature detection with application of signal detection methodology. Perception \& Psychophysics, 1977, 21, 88-92.

YIN, R. Looking at upside down faces. Journal of Experimental Psychology, 1969, 81, 141-145.

(Received for publication June 4, 1979; revision accepted October $2,1979$. 